\title{
Drain site radiotherapy in malignant pleural mesothelioma: a wasted resource
}

\section{To the Editors:}

Prophylactic drain site irradiation is currently used to prevent tumour nodule formation in pleural mesothelioma. We present a case which highlights the inadequacy of this practice and discuss the recent findings of O'ROURKE et al. [1], which suggest irradiation should only be offered for symptomatic tumour nodules.

In February 2003, a 71-yr-old male presented with breathlessness on exertion associated with loss of appetite. A chest radiograph demonstrated left pleural effusion. Aspiration of the fluid with pleural biopsy was performed, yielding a histological diagnosis of malignant pleural mesothelioma. The patient was referred for consideration of irradiation to the chest drain site and received the standard $21 \mathrm{~Gy}$ in three fractions, using $250 \mathrm{kV}$ photons, on consecutive days. The radiotherapy field was a standard $6-\mathrm{cm}$ circle, to allow a margin around the drain site of $>2 \mathrm{~cm}$ in all directions. The drain site was in the left mid-axillary line. Radiotherapy was completed in March 2003.

The patient remained well and asymptomatic, but in June 2003 developed a subcutaneous tumour deposit in the chest wall, at the superior border of the previous radiotherapy field. Initially this was asymptomatic and was therefore observed; however, within 1 month it had become more uncomfortable. The tumour deposit measured $5 \mathrm{~cm}$ of diameter and was by then overlapping with the previous treatment field. Re-treatment with radiotherapy was administered: the field was a 9-cm circle (to allow 2-cm margin again) and a dose of 30 Gy was delivered in 10 fractions over 2 weeks using $12 \mathrm{MeV}$ electrons prescribed at surface.

Following treatment there was complete resolution of the tumour deposit and the patient remained well and symptom free. The patient had no further problems with subcutaneous tumour before his death in July 2004.

Patients with mesothelioma usually require pleural aspiration, pleural biopsy or thoracoscopy during diagnosis. Following these procedures there is a recognised risk of malignant seeding along the track of the needle or trocar, causing subcutaneous nodule formation. The standard of care has been to offer prophylactic irradiation following these procedures; however, the evidence that this reduces nodule formation or improves quality of life is questionable. Two studies [2,3] are the basis for our current practice. Low et al. [2] reviewed 20 patients who had received radiotherapy to 38 sites. During follow-up of 1-10 months, none of these patients developed tumour seeding. Boutin et al. [3] randomised 40 patients to radiotherapy or nonradiotherapy after thoracoscopy. None of the patients who received radiotherapy developed nodules, whereas $40 \%$ of patients who did not receive radiotherapy developed nodules. On the basis of these studies, international standard practice has been to offer prophylactic irradiation to intervention sites in mesothelioma.

O'ROURKE et al. [1] have recently published a trial involving 61 patients randomised to standard radiotherapy versus noradiotherapy. Of these, 12 developed nodules: eight in the radiotherapy arm and four in the control arm. There was no statistically significant difference in risk of subcutaneous tumour nodule development between the two arms. The study also highlighted that radiotherapy is not without morbidity. Approximately $35 \%$ of patients assessed found that radiotherapy affected energy levels, to different degrees. Only three of the 12 nodules were tender or troublesome. In view of this more robust trial with significantly more patients than previous studies, we suggest irradiation is indicated only in those with symptomatic tumour nodules.

\section{R. Muirhead and N. O'Rourke}

The Beatson West of Scotland Cancer Centre, Gartnavel General Hospital, Glasgow, UK.

\section{STATEMENT OF INTEREST}

None declared.

\section{REFERENCES}

1 O'Rourke N, Garcia JC, Paul J, Lawless C, McMenemin R, Hill J. A randomised controlled trial of intervention site radiotherapy in malignant pleural mesothelioma. Radiother Oncol 2007; 84: 18-22.

2 Low EM, Khoury GG, Matthews AW, Neville E. Prevention of tumour seeding following thoracoscopy in mesothelioma by prophylactic radiotherapy. Clin Oncol (R Coll Radiol) 1995; 7: 317-318.

3 Boutin C, Rey F, Viallat JR. Prevention of malignant seeding after invasive diagnostic procedures in patients with pleural mesothelioma. A randomized trial of local radiotherapy. Chest 1995; 108: 754-758. 